\title{
Investigation of fascial treatment effectiveness on pain, flexibility, functional level, and kinesiophobia in patients with chronic low back pain
}

\author{
DOI: https://doi.org/10.5114/pq.2019.86461
}

\author{
Doğan Burak Endamlı', Kezban Bayramlar², Begümhan Turhan² \\ ${ }^{1}$ Alleben Medical Center, Gaziantep, Turkey \\ ${ }^{2}$ Department of Physiotherapy and Rehabilitation, Faculty of Health Sciences, Hasan Kalyoncu University, Gaziantep, \\ Turkey
}

Abstract

Introduction. Fascial manipulations have received attention in the treatment of chronic low back pain (CLBP). The purpose of this study is to investigate the effectiveness of fascial manipulation on pain, flexibility, functional level, and kinesiophobia in patients with CLBP.

Methods. Overall, 55 individuals with a history of CLBP, without neurological deficits, lumbar disc herniation, lumbarization, spondylosis, or lumbar surgical history were included in the study. The participants were randomly divided into 2 groups depending on the patient protocol numbers. Subjects with odd protocol numbers were assigned to the study group (fascial manipulation) and those with even numbers to the control group (conventional physiotherapy). Both groups were assessed before and after the treatment in terms of pain, flexibility, functional level, and kinesiophobia.

Results. There were significant differences after both treatment methods in terms of pain, functional level, and flexibility $(p<0.05)$. However, pain levels decreased more statistically significantly in the fascial manipulation group than in the control group $(p<0.05)$. No significant difference was observed between the groups in terms of functional level, flexibility, or kinesiophobia after the treatment $(p>0.05)$.

Conclusions. We stated that fascial manipulation was more effective on pain than conventional physiotherapy methods (hot pack, microwave diathermy, interferential flow-vacuum applications, posterior pelvic tilt and abdominal exercises, stretching hip flexors and lumbar extensors) in patients with CLBP.

Key words: fascia, fascial manipulation, low back pain

\section{Introduction}

Chronic low back pain (CLBP) is a disease which causes significant disability, leading to health costs and loss of working days. CLBP may be associated with thoracolumbar fascia (TLF) pathologies because of fascial anatomical pathways. Therefore, the recent approaches to fascial manipulation in the thoracolumbar region have received attention in the treatment of CLBP [1].

Fascia is a layer of connective tissue that consists of irregular collagenous fibres and surrounds structures perfectly [2, 3]. Deep fascia surrounds muscles, bones, nerves, and blood vessels. It is very rich in hyaluronic acid $(\mathrm{HA})$, also called hyaluronan. HA has a role as a slippery liquid in the sliding of deep fascia. When HA levels decrease, local slippery properties of the tissues become reduced, and pain is observed due to the loss of fascial movements. The main aim of fascial manipulation is to increase the fluidity of fascia depending on HA heating [2, 4-7]. The functionality of fascia can be impaired because of microtraumas, physical trauma, or inflammation. Impaired fascia disturbs the normal biomechanics of the body and causes pain. Fascial manipulations are clinically effective for reducing pain and improving physiological functions in somatic disorders [8]. Also, fascia is rich in mechanoreceptors, which are very sensitive to manual pressures. There are also smooth muscle cells in the fascia. These are innervated by the autonomic nervous system. It is thought that the stimulation of intrafascial sympathetic afferents by fascial manipulation can alter the local autonomic nervous system, as well as the local circulation and the cell matrix density [2, 9].

The lumbar region plays an important role in the postural stability of the body. A complex myofascial network is formed around the trunk to stabilize the lumbar spine. The central point of this fascial network in the lumbar region is TLF, located around the paraspinal muscles and the sacral region. The paraspinal muscles, surrounded by this fascia, are connected to the spinous process. TLF goes to the anterior wall of the abdomen and surrounds the transversus abdominis muscle, obliquus internus abdominis muscle, and obliquus externus abdominis muscle; then it joins the linea alba. TLF joins the gluteal fascia via gluteus maximus and biceps femoris muscles and contacts the lower extremity. This deep fascia extends to the $12^{\text {th }}$ rib. TLF is continuous in the thoracic and cervical region, and eventually inserts to the skull base. Many muscles of trunk and limbs are attached to the connective tissue of TLF and modulated by the tension and stiffness of this deep fascia. Fascia can be associated with the pathophysiology of CLBP because of the relationship between CLBP and fascial tissues [9, 10].

Today, electrotherapy, superficial and deep warm treatments, strengthening and stretching exercises for back and abdominal muscles are frequently used in physiotherapy approaches for CLBP. In recent years, manipulative thera-

Correspondence address: Begümhan Turhan, Hasan Kalyoncu University, Department of Physiotherapy and Rehabilitation, Havaalanı 8.km, Gaziantep, Turkey, e-mail: begum.aliosmanoglu@hku.edu.tr

Received: 08.04.2019

Accepted: 20.05.2019

Citation: Endamlı DB, Bayramlar K, Turhan B. Investigation of fascial treatment effectiveness on pain, flexibility, functional level, and kinesiophobia in patients with chronic low back pain. Physiother Quart. 2019;27(3):1-5; doi: https://doi.org/10.5114/pq.2019.86461. 
pies have come into prominence with studies aimed to relax the muscles around the spine. The hypothesis of this study is that fascial manipulation is effective on pain, flexibility, kinesiophobia, and functional level in patients suffering from CLBP. The study was conducted to investigate the effects of fascial manipulation in patients with CLBP.

\section{Subjects and methods}

All participants were informed about the purpose of the study, treatment, duration of treatment, and assessments. The study was designed as a randomized controlled trial. It was carried out in the Alleben Medical Center, Gaziantep. The 55 participants were randomly divided into 2 groups depending on the patient protocol numbers. Patients with odd protocol numbers were assigned to the treatment group and those with even numbers to the control group. The subjects were aged 20-60 years, had a history of CLBP (experienced pain for more than 3 months) and no neurological deficit, lumbar disc herniation, lumbarization, spondylosis, or lumbar surgical history.

Pre-treatment pain, functional level, flexibility, and kinesiophobia were evaluated in both groups. Visual analogue scale (VAS) was used to determine the level of pain. The flexibility of the lumbar region was assessed in a sitting position with the sit and reach test [11]. The functional level was evaluated with the Oswestry Low Back Pain Disability Questionnaire [12]. The Turkish version of the Tampa Scale for Kinesiophobia (TSK), a valid and reliable tool, was used to determine the kinesiophobia levels [13].

Fascial manipulation techniques (Figures 1-5) were applied to the study group $(n=28)$ in 5 stages after a conventional physiotherapy protocol. The control group $(n=27)$ only received the conventional physiotherapy protocol. The fascial manipulation techniques were selected from Bowstring's fascial release techniques and are detailed below [14].

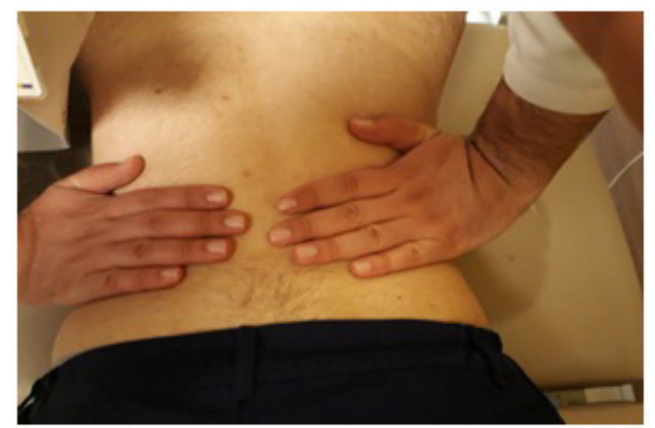

Figure 1. Thoracolumbar relaxation technique application while the patient is prone and the therapist's hands are horizontally opposite positioned on the thoracolumbar fascia

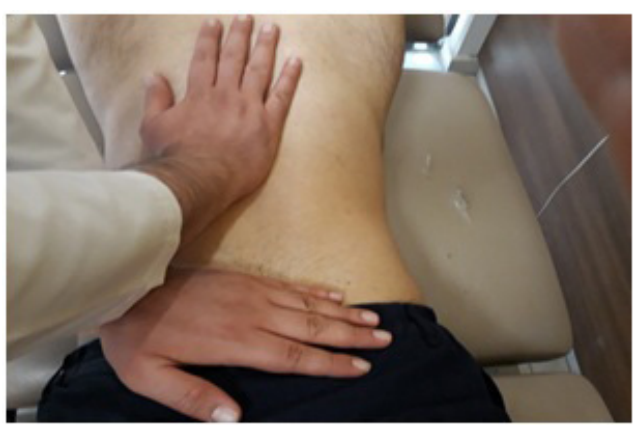

Figure 2. Sacrum technique application while the patient is prone and the therapist is standing behind the patient. The therapist's left hand is horizontally on sacrum, the other hand is obliquely and horizontally positioned on T7-T12
1. The patient lay in prone position for the thoracolumbar relaxation technique. As the therapist was standing on one side of the patient, the hand was placed on both sides of the spinous attachment sites of TLF in the lumbar area. Hands were removed from each other and the technique was applied softly (Figure 1).

2. The patient was prone for the sacrum technique. The therapist stood beside the patient, the hands were crosswise, one hand on sacrum and the other on L1. Then the tissues were stretched to the opposite side (Figure 2).

3. Tractus iliotibialis was palpated and tension region was found for the tractus iliotibialis technique. Two thumbs were overturned and a strong pressure was applied in the

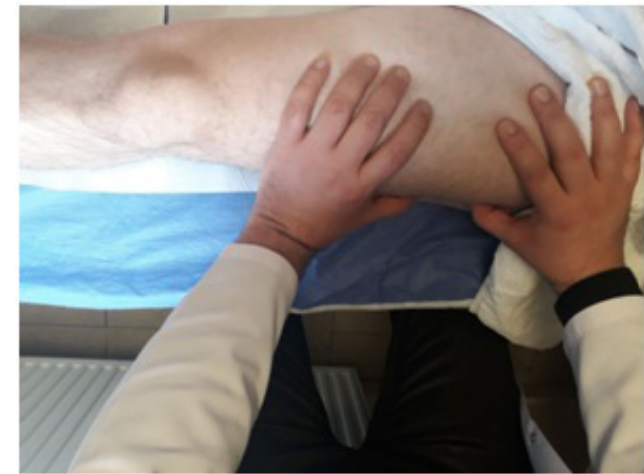

Figure 3. lliotibial tract technique application while the patient is supine and the therapist is sitting behind the patient.

The therapist places the thumbs of two hands in the most taut place of tensor fasciae latae. In this position, they press their fingers towards the bed

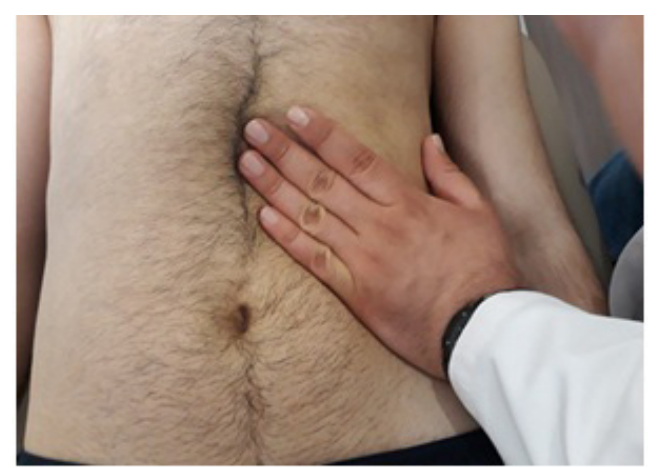

Figure 4. Triangulare sinistrum technique application while the patient is supine and the therapist is standing on the left side of the patient. The therapist's left hand's index finger is on the xiphoid process. The hand is moved with the skin towards the xiphoid process

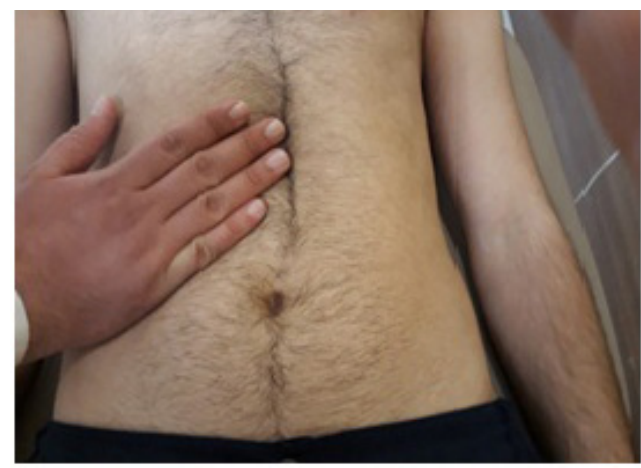

Figure 5. Teres hepatis technique application while the patient is supine and the therapist is standing on the right side of patient. The therapist's left hand's index finger is on the xiphoid process. The hand is moved with the skin towards the xiphoid process 
posteromedial direction until the tissue relaxed. This technique was applied unilaterally (Figure 3).

4. The patient's position was supine and the therapist stood by the patient for triangulare sinistrum technique. The equilibrium point was found by applying pressure to the left of the epigastric region. The tension area was detected by moving the fingers laterally. The finger was held on the tension area until the tension disappeared (Figure 4).

5. For the teres hepatis technique, the patient and the therapist took the same positions as in the triangulare sinistrum technique. The same application was performed on the right side (Figure 5).

Mobilization techniques were applied during $120 \mathrm{sec}-$ onds for each technique and the treatment took 10 minutes overall. Conventional physiotherapy treatment was performed for 5 days per week, as a total of 15 sessions. In the control group, patients received hot pack, microwave diathermy, interferential flow-vacuum application, as well as posterior pelvic tilt and abdominal exercises, and hip flexor and lumbar extensor muscles stretching exercises. In the study group, 5 sessions of fascial manipulation, once every 3 days, were added to the conventional treatment. During the study, all the evaluations were performed by another physical therapist, who was unaware of the treatment and the research. After the treatment, patients were re-evaluated and the results were compared with the pre-treatment values.

\section{Statistical analysis}

The sample size of 30 patients was calculated to yield $95 \%$ power at $p=0.05$. The Statistical Package for the Social Sciences (SPSS) software (version 20 for Windows) was applied for data analysis. The value of $p=0.05$ was considered significant. Shapiro-Wilk test for normality was used. The values were displayed as means and standard deviations $(\bar{x} \pm S D)$. Independent sample $t$-test served to examine differences between the initial values of groups. A two-way analysis of variance and paired $t$-tests were applied to test for differences between and within groups.

\section{Ethical approval}

The research related to human use has complied with all the relevant national regulations and institutional policies, has followed the tenets of the Declaration of Helsinki, and has been approved by the Research Ethics Committee of the Faculty of Health Sciences, Hasan Kalyoncu University (decision No. 2016-06).

\section{Informed consent}

Informed consent has been obtained from all individuals included in this study.

\section{Results}

The mean age of the study group was $37.14 \pm 11.56$ years, the mean height was $172.29 \pm 10.05 \mathrm{~cm}$, the mean body weight was $77.75 \pm 11.81 \mathrm{~kg}$, and the mean body mass index (BMI) was $26.27 \pm 3.84 \mathrm{~kg} / \mathrm{m}^{2}$. The mean age, height, weight, and $\mathrm{BMl}$ in the control group were $37.93 \pm$ 10.87 years, $171.07 \pm 8.65 \mathrm{~cm}, 75.22 \pm 17.10 \mathrm{~kg}$, and $25.48 \pm$ $4.28 \mathrm{~kg} / \mathrm{m}^{2}$, respectively. The participants were similar in terms of their height, weight, BMI, and pre-treatment scores $(p>0.05)$ (Table 1).

Among the individuals in the study group, improvements were observed in pain, flexibility, and functional levels after treatment $(p<0.05)$. A significant difference was found in pain, flexibility (sit and reach test), and functional levels when the control group was examined before and after treatment $(p<0.05)$ (Table 2).

There was a significant decrease in pain in the study group after treatment when the groups were compared in terms of pain $(p<0.05)$. When the groups were examined for flexibility, no significant difference was observed after treatment in terms of sit and reach test $(p>0.05)$. With re-

Table 2. Comparison of pre- and post-treatment results within groups in terms of the evaluated parameters

\begin{tabular}{|l|c|c|c|c|}
\hline & $\begin{array}{c}\text { SG } \\
\text { (pre/ } \\
\text { post) }\end{array}$ & $p$ & $\begin{array}{c}\text { CG } \\
\text { (pre/ } \\
\text { post) }\end{array}$ & $p$ \\
\hline Pain & -4.138 & $0.001^{*}$ & -3.280 & $0.001^{*}$ \\
\hline Functional level & -4.017 & $0.001^{*}$ & -2.742 & $0.006^{*}$ \\
\hline Kinesiophobia & -0.785 & 0.432 & -0.285 & 0.776 \\
\hline Sit and reach test (flexibility) & -4.458 & $0.001^{*}$ & -3.528 & $0.002^{*}$ \\
\hline
\end{tabular}

${ }^{*} p<0.05$, statistically significant

SG - study group, CG - control group, pre - pre-treatment, post post-treatment

Table 3. Post-treatment results within groups in terms of the evaluated parameters

\begin{tabular}{|l|r|r|c|}
\hline Parameters & \multicolumn{1}{c|}{ SG (mean) } & CG (mean) & $p$ \\
\hline Pain & $3.79 \pm 1.73$ & $4.85 \pm 1.94$ & $0.012^{*}$ \\
\hline Sit and reach test & $-6.86 \pm 12.66$ & $-5.57 \pm 19.77$ & 0.245 \\
\hline Functional level & $13.82 \pm 7.49$ & $16.26 \pm 7.90$ & 0.080 \\
\hline Kinesiophobia & $42.14 \pm 8.43$ & $42.89 \pm 5.18$ & 0.437 \\
\hline
\end{tabular}

${ }^{*} p<0.05$, statistically significant

SG - study group, CG - control group

Table 1. Participants' demographic data and pre-treatment scores

\begin{tabular}{|c|c|c|c|c|}
\hline Characteristics & $\mathrm{SG}(n=28)$ & $\mathrm{CG}(n=27)$ & $t$ & $p$ \\
\hline Age (years) & $37.14 \pm 11.56$ & $37.93 \pm 10.87$ & -0.259 & 0.797 \\
\hline Height (cm) & $172.29 \pm 10.05$ & $171.07 \pm 8.65$ & 0.478 & 0.624 \\
\hline Weight (kg) & $77.75 \pm 11.81$ & $75.22 \pm 17.10$ & 0.640 & 0.525 \\
\hline BMI $\left(\mathrm{kg} / \mathrm{m}^{2}\right)$ & $26.27 \pm 3.84$ & $25.48 \pm 4.28$ & 0.715 & 0.478 \\
\hline Pain (VAS) & $6.29 \pm 1.76$ & $6.26 \pm 1.93$ & -0.085 & 0.932 \\
\hline Sit and reach test $(\mathrm{cm})$ & $-11.54 \pm 15.01$ & $-9.54 \pm 20.97$ & -0.408 & 0.685 \\
\hline Functional level score & $21.71 \pm 7.32$ & $21.59 \pm 8.70$ & -0.042 & 0.966 \\
\hline Kinesiophobia score & $43.39 \pm 7.53$ & $43.52 \pm 4.34$ & -0.220 & 0.826 \\
\hline
\end{tabular}

SG - study group, CG - control group, BMI - body mass index, VAS - visual analogue scale 
gard to functional level, there was no significant difference between the post-treatment outcomes $(p>0.05)$. The kinesiophobia scores were similar between the groups after treatment $(p>0.05)$ (Table 3).

\section{Discussion}

Many treatments have been described for CLBP, but there is not any proven therapy protocol. In the recent years, manipulative therapy techniques have received attention and studies have started to increase in the literature. In our study, we examined the efficiency of fascial manipulation in patients with CLBP.

Inactivity due to CLBP results in abnormal changes of the fascial tissue. Active and passive movements are affected because of pain and this reduces the range of motion. After fascial manipulation, recovery of symptoms is often seen [8]. Bordoni and Zanier [15] examined the symptoms and systemic linkages of the skin and the fascia. It turned out that fascia, which embryologically originates from the mesodermal layer, can transmit the effects of traumas between parts of the body. Today's physical therapy and rehabilitation treatment approaches usually appear to be results of a local evaluation of the body, not as a whole. In cases with CLBP, if the disorder originates from the disc or muscle spasm, the local treatments of this region solve the problem temporarily only.

In the recent years, TLF has attracted attention as a structure that creates potential pain in the dorsal part of body. Increased thickness and echogenicity were observed in the perimuscular connective tissues of TLF among individuals who had suffered from CLBP for more than 12 months [16]. Abnormal connective tissue structure may be a factor predisposing to CLBP. In addition, the fibrous tissue that develops as a result of trauma and adhesions may also lead to abnormal connective tissue structure [17]. A study using ultrasound images revealed that in individuals with CLBP, TLF was thicker than in normal subjects [18].

Studies on the effectiveness of fascial mobilization are rare. Branchini et al. [19] compared exercise and manual therapy with fascial mobilization therapy. They observed that there was a further decrease in pain, especially in the group treated by fascial mobilization. Day et al. [7] studied 28 patients with shoulder pain and found out that the pain of patients treated by fascial mobilization technique was significantly reduced in accordance with VAS. Guarda-Nardini et al. [20] compared botulinum toxin injection treatment with fascial mobilization technique among 30 individuals with myofascial pain of jaw. They found that a single-dose botulinum toxin injection was more effective for jaw movements than the fascial technique, but the treatments had similar effects on pain. A study on cyclists with hip and inguinal pain reported that fascial mobilization techniques relaxed the pelvic floor muscles and reduced the pain [21]. Fascial manipulation results in local vasodilatation, increased tissue viscosity, and loose muscle tissue $[22,23]$. During fascial manipulation, the pressure in the fluid between the muscle and the fascia causes an increase of the fluid gap. As a result, the thickness between the 2 fascial layers increases. The presence of a thicker fluid gap can improve the sliding system and allow the muscles to work more efficiently [24]. The relaxation of fascial tissue affects not only itself but also the vascular system, neural networks, and immune cells within the fascial tissue [25]. Impulses generated by fascial manipulation provide stimulation of intrafascial mechanoreceptors, and parasympathetic responses are produced as a result. The increasing mobility of the fascia with fascial manipulation reduces pain [19].
These findings support the pain-related results of our study. Also, the study showed that fascial manipulation was more effective than the conventional physiotherapy treatment protocol in reducing pain in patients with CLBP.

There are 3 manual therapy motions described in fascial manipulation applications. These are constant sliding, perpendicular vibration, and tangential oscillation. Roman et al. [24] compared various manual therapy techniques on the fluidity of $\mathrm{HA}$ in the fascia. They suggested that vibration and sliding techniques increased the HA fluidity the most. However, more studies are needed in which all the techniques would be applied or compared. In the presented study, we performed only myofascial release (constant sliding technique), which is a soft tissue technique and is frequently used in physical therapy [26, 27].

In fascial techniques, a little load must be applied. The practitioner uses pressure until to feel a barrier that resists the fascia. The sliding continues for 90-120 seconds without tissue strain [23]. We preferred the application time of 120 seconds for each technique.

In this study, there was a decrease in the functional level scores and flexibility after the treatment, but there was no statistically significant difference between the groups. It is expected that the functional level could not show any difference in short-term treatment. Also, flexibility was similar as a result of both treatment methods. A change in flexibility in both groups could be expected owing to the decrease in pain. We believe that a re-evaluation of individuals involved in this study after a certain period could reveal a meaningful difference in the functional level.

TSK is a scale that evaluates the fear associated with movement and pain. It helps estimate the disability and pain severity rates due to the low back pain; it is also used in the assessment of fear of movement before rehabilitation [28, 29]. A randomized controlled trial including a multidisciplinary treatment program for 13 months of follow-up, exercise, and education showed a significant decrease in kinesiophobia and pain, as well as an increase in quality of life [30]. In our study, pre- and post-treatment TSK scores of the individuals were significantly different within the groups and there was no significant difference between the groups. We think that kinesiophobia would heal more with exercise. In our study, the lack of change in kinesiophobia could be a result of the local exercises that were applied only for the lumbar region.

After treatment, the significant difference in pain between the groups showed that the fascial manipulation might be tried as a treatment method of pain relief for patients with CLBP. The results obtained in this study emphasize the importance of adding manipulative treatments, which are less costly than other treatment methods, to therapeutic protocols.

\section{Limitations}

The individuals in the study and control groups could not be followed up for a long time. Long-term changes should be investigated after fascial manipulation. As the functional level and kinesiophobia may change with exercise, the absence of an exercise group could be considered as a limitation.

\section{Conclusions}

Our study contributes to the literature on manipulative treatment. Fascial manipulation techniques turned out more effective on pain than conventional physiotherapy methods in patients with CLBP. 


\section{Disclosure statement}

No author has any financial interest or received any financial benefit from this research.

\section{Conflict of interest}

The authors state no conflict of interest.

\section{References}

1. Van Dieën JH, Selen LP, Cholewicki J. Trunk muscle activation in low-back pain patients, an analysis of the literature. J Electromyogr Kinesiol. 2003;13(4):333-351; doi: 10.1016/S1050-6411(03)00041-5.

2. Bordoni B, Zanier E. Clinical and symptomatological reflections: the fascial system. J Multidiscip Healthc. 2014; 7:401-411; doi: 10.2147/JMDH.S68308.

3. Tozzi P. Selected fascial aspects of osteopathic practice. J Bodyw Mov Ther. 2012;16(4):503-519; doi: 10.1016/j.jbmt.2012.02.003.

4. Adstrum S, Hedley G, Schleip R, Stecco C, Yucesoy CA. Defining the fascial system. J Bodyw Mov Ther. 2017; 21(1):173-177; doi: 10.1016/j.jbmt.2016.11.003.

5. Pavan PG, Stecco A, Stern R, Stecco C. Painful connections: densification versus fibrosis of fascia. Curr Pain Headache Rep. 2014;18(8):441; doi: 10.1007/s11916014-0441-4.

6. Stecco A, Gesi M, Stecco C, Stern R. Fascial components of the myofascial pain syndrome. Curr Pain Headache Rep. 2013;17(8):352; doi: 10.1007/s11916-0130352-9.

7. Day JA, Stecco C, Stecco A. Application of Fascial Manipulation ${ }^{\odot}$ technique in chronic shoulder pain - anatomical basis and clinical implications. J Bodyw Mov Ther. 2009;13(2):128-135; doi: 10.1016/j.jbmt.2008.04.044.

8. Meltzer KR, Cao TV, Schad JF, King H, Stoll ST, Standley PR. In vitro modeling of repetitive motion injury and myofascial release. J Bodyw Mov Ther. 2010;14(2):162171; doi: 10.1016/j.jbmt.2010.01.002.

9. Willard FH, Vleeming A, Schuenke MD, Danneels L, Schleip R. The thoracolumbar fascia: anatomy, function and clinical considerations. J Anat. 2012;221(6):507-536; doi: 10.1111/j.1469-7580.2012.01511.x.

10. Stecco C, Gagey O, Macchi V, Porzionato A, De Caro R, Aldegheri $\mathrm{R}$, et al. Tendinous muscular insertions onto the deep fascia of the upper limb. First part: anatomical study. Morphologie. 2007;91(292):29-37; doi: 10.1016/ j.morpho.2007.05.001.

11. Hui SSC, Yuen PY. Validity of the modified back-saver sitand-reach test: a comparison with other protocols. Med Sci Sports Exerc. 2000;32(9):1655-1659; doi: 10.1097/ 00005768-200009000-00021.

12. Yakut E, Düger T, Öksüz Ç, Yörükan S, Üreten K, Turan D, et al. Validation of the Turkish version of the Oswestry Disability Index for patients with low back pain. Spine. 2004;29(5):581-585; doi: 10.1097/01.BRS.0000113869. 13209.03

13. Tunca Yilmaz Ö, Yakut Y, Uygur F, Uluğ N. Turkish version of the Tampa Scale for Kinesiophobia and its testretest reliability [in Turkish]. Fizyoter Rehabil. 2011; 22(1):44-49.

14. Speece CA, Crow WT, Simmons SL. Osteopathic techniques by W.G. Sutherland: ligamentous articular strain (LAS) [in German]. Stuttgart: Hippokrates; 2003.

15. Bordoni B, Zanier E. Skin, fascias, and scars: symptoms and systemic connections. J Multidiscip Healthc. 2014;7: 11-24; doi: 10.2147/JMDH.S52870.
16. Taguchi T, Hoheisel U, Mense S. Dorsal horn neurons having input from low back structures in rats. Pain. 2008; 138(1):119-129; doi: 10.1016/j.pain.2007.11.015.

17. Langevin HM, Fox JR, Koptiuch C, Badger GJ, Greenan-Naumann AC, Bouffard NA, et al. Reduced thoracolumbar fascia shear strain in human chronic low back pain. BMC Musculoskelet Disord. 2011;12:203; doi: 10.1186/1471-2474-12-203.

18. Langevin HM, Stevens-Tuttle D, Fox JR, Badger GJ, Bouffard NA, Krag MH, et al. Ultrasound evidence of altered lumbar connective tissue structure in human subjects with chronic low back pain. BMC Musculoskelet Disord. 2009;10:151; doi: 10.1186/1471-247410-151.

19. Branchini M, Lopopolo F, Andreoli E, Loreti I, Marchand AM, Stecco A. Fascial Manipulation ${ }^{\circledR}$ for chronic aspecific low back pain: a single blinded randomized controlled trial. F1000Res. 2015;4:1208; doi: 10.12688/ f1000research.6890.2.

20. Guarda-Nardini L, Stecco A, Stecco C, Masiero S, Manfredini D. Myofascial pain of the jaw muscles: comparison of short-term effectiveness of botulinum toxin injections and fascial manipulation technique. Cranio. 2012;30(2): 95-102; doi: 10.1179/crn.2012.014.

21. Navot S, Kalichman L. Hip and groin pain in a cyclist resolved after performing a pelvic floor fascial mobilization. J Bodyw Mov Ther. 2016;20(3):604-609; doi: 10.1016/j.jbmt.2016.04.005.

22. Schleip R, Naylor IL, Ursu D, Melzer W, Zorn A, Wilke HJ, et al. Passive muscle stiffness may be influenced by active contractility of intramuscular connective tissue. Med Hypotheses. 2006;66(1):66-71; doi: 10.1016/j. mehy.2005.08.025.

23. Tozzi P. Does fascia hold memories? J Bodyw Mov Ther.2014;18(2):259-265;doi:10.1016/j.jbmt.2013.11.010.

24. Roman M, Chaudhry H, Bukiet B, Stecco A, Findley TW. Mathematical analysis of the flow of hyaluronic acid around fascia during manual therapy motions. J Am Osteopath Assoc. 2013;113(8):600-610; doi: 10.7556/ jaoa.2013.021.

25. Langevin HM, Bouffard NA, Fox JR, Palmer BM, Wu J, latridis $\mathrm{JC}$, et al. Fibroblast cytoskeletal remodeling contributes to connective tissue tension. J Cell Physiol. 2011; 226(5):1166-1175; doi: 10.1002/jcp.22442.

26. Hamoda RE, Osman DA, Hamada HA, Radwan R, Yousef AM, Samea GAA, et al. Effect of myofascial release on electrophysiological and clinical measures of pregnant women with carpal tunnel syndrome. Physiother Quart. 2019;27(1):18-24; doi: 10.5114/pq.2019.83057.

27. Wilke J, Vogt L, Banzer W. Immediate effects of self-myofascial release on latent trigger point sensitivity: a randomized, placebo-controlled trial. Biol Sport. 2018;35(4) 349-354; doi: 10.5114/biolsport.2018.78055.

28. Kori SH, Miller RP, Todd DD. Kinesophobia: a new view of chronic pain behaviour. Pain Manag. 1990;3:35-43.

29. Swinkels-Meewisse EJ, Swinkels RA, Verbeek AL, Vlaeyen JW. Oostendorp R.A. Psychometric properties of the Tampa Scale for Kinesiophobia and the fear-avoidance beliefs questionnaire in acute low back pain. Man Ther. 2003;8(1):29-36; doi: 10.1054/math.2002.484.

30. Monticone M, Ambrosini E, Rocca B, Magni S, Brivio F, Ferrante S. A multidisciplinary rehabilitation programme improves disability, kinesiophobia and walking ability in subjects with chronic low back pain: results of a randomised controlled pilot study. Eur Spine J. 2014; 23(10):2105-2113; doi: 10.1007/s00586-014-3478-5. 